\title{
Technical Research on Advanced Computing
}

\author{
Hong Yang ${ }^{1}$, Shurui Zhang ${ }^{2}$, Xiong Guo ${ }^{3}$ and Mengliang $\mathrm{Li}^{4, *}$ \\ 1,2,3,4 China Electronics Standardization Institute, China \\ *Corresponding author
}

\begin{abstract}
Due to the wide application of Internet of things, the real-time of data and the rapid development of $\mathrm{AI}$, advance computing can provide technical support and guarantee. Firstly, this paper introduces the background, basic concept and development history, and classification of advanced computing. Secondly, the concepts, characteristics and key technologies of edge computing \& fog computing, quantum computing, cognitive computing and ubiquitous computing are mainly introduced. Thirdly, the industrial application of advance computing are emphasized. Finally, the suggestion of advance computing is given.
\end{abstract}

Keywords-advanced computing; basic concepts; industrial application; suggestion

\section{INTRODUCTION}

\section{A. Background}

At present, the new technological revolution and industrial transformation are rising, and the global industrial technology system, development mode and competition pattern are facing major changes. The developed countries have introduced "reindustrialization" national strategies with advanced manufacturing as the core. As China puts forward the implementation of "China made 2025", "one area one road" and "millions of industrial APP cultivation" strategy, the demand for computing technology is getting higher and higher, not only to be fast, but also to calculate well and steadily.

In the face of the continuous production of heterogeneous mass data, the traditional computing technology has gradually become tired. In recent years, international semiconductor technology has increasingly approached the physical limit in material and manufacturing technology. The development of traditional computing technology is faced with systemic difficulties. Advanced computing technology came into being to solve problems of different levels, different angles and different application scenes. At the same time, Application of Internet of Everything, Real-time and security of data and Artificial intelligence and deep learning require more advanced computation.

\section{B. Concept and Development History of Advanced Computing}

\section{1) Concept}

Advanced computing is to build the next generation of information infrastructure by using high-performance supercomputers and network systems, which makes the computers of dispersion in different geographical locations a huge virtual supercomputer and achieves the interconnection and sharing of network resources.

\section{2) Development history of advanced computing}

The history of advanced computing is relatively long and continues to develop [1]. According to the change of computing speed capability, the development history of advanced computing has gone through three major stages: device change, architecture change and computer principle change. Quantum computing, cloud computing, fog computing and edge computing began in 1988, 2006, 2011 and 2014 respectively.

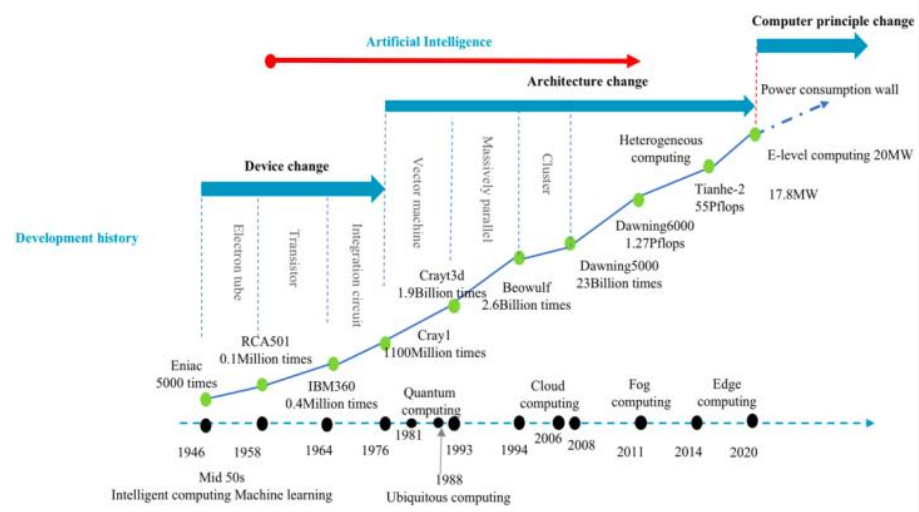

FIGURE I. DEVELOPMENT HISTORY OF ADVANCED COMPUTING

\section{Classification of Advanced Computing}

Advanced computing includes edge computing and fog computing, quantum computing, ubiquitous computing, and cognitive computing. Among them, edge computing and fog computing are discussed from the point of view of distributed computing. Quantum computing is classified from the angle of quantum superposition. Ubiquitous computing is from the perspective of customer experience, and cognitive computing is from the angle of artificial intelligence [2].

\section{EDGE COMPUTING \& Fog COMPUTING}

\section{A. Edge Computing}

\section{1) Concepts}

Edge computing is performed on an open platform at the network edge near things or data sources, integrating network, computing, storage, and application core capabilities and providing edge intelligent services. Edge computing open platform includes intelligent asset, intelligent gateway, intelligent system and intelligent service. Edge computing can be contrasted with centralized computing (for example, cloud 
computing), where the resources are centralized in large remote data centers. However, it is important to note that edge computing is complementary to centralized forms of computing such as cloud computing and that in any given system, edge computing is typically used in conjunction with centralized forms of computing such as cloud computing [3].

\section{2) Reference architecture}

The reference architecture of edge computing includes application domain, data domain, network domain and equipment domain.

a) Application domain: Implement EC Industry application and support EC service operation.

b) Data domain: Provide life cycle service, enable data security \& privacy, support distributed computing, and scalability of ECN resource.

c) Network domain: Provide connection service for interconnected systems, data aggregation and bearing; Satisfy real-time requirement of service through real-time connection and transmission.

d) Equipment domain: Support real-time intelligent interconnection and intelligent application of field equipment; Heterogeneous system architecture can meet the requirement of real-time service and intelligent equipment; Lightweight system architecture enable low power consumption.

\section{3) Key technologies}

The key technologies of edge computing include Software Defined Connection, Time Sensitive Network and Heterogeneous Computing.

\section{a) Software defined connection}

- Automated operation and maintenance: unified management of Connection, terminal, application ;

- Unified policy of the network, data transport and security;

- Quick development, deployment and usage of application of machine vision, industrial control, etc.

b) Time sensitive network

- A set of standards under development by the TSN task group of the IEEE 802.1 working group;

- Built on the MAC/PHY layer of Ethernet, offers a low latency, time deterministic and high reliable way to send time-critical traffic over standard Ethernet infrastructures;

\section{c) Heterogeneous computing}

- Build a new system by using different types of instruction sets and architectural computing units

- Utilize the advantages of various technical units to meet the requirement of real-time service and high performance

B. Fog Computing

1) Concepts
A term created by Cisco that refers to extending cloud computing to the edge of an enterprise's network. Also known as Edge Computing or fogging, fog computing facilitates the operation of compute, storage, and networking services between end devices and cloud computing data centers. While edge computing is typically referred to the location where services are instantiated, fog computing implies distribution of the communication, computation, and storage resources and services on or close to devices and systems in the control of end-users. Fog computing is a medium weight and intermediate level of computing power. The main features of fog computing include response feedback delay low, wide distribution and high density, support local real-time processing, support the movement of intelligent terminals and support wireless fast access [4].

\section{2) Reference architecture}

The reference architecture of fog computing includes performance, security, manageability, data analytics and control, IT Business and cross fog applications.

e) Performance: Low latency is one of the driving reasons to adopt fog architectures.

f) Security: End-to-end security is critical to the success of all fog computing deployment scenarios.

g) Manageability: Managing all aspects of fog deployments, which include RAS, DevOps, etc., is a critical aspect across all layers of a fog computing hierarchy.

h) Data Analytics and Control: The ability for fog nodes to be autonomous requires localized data analytics coupled with control.

i) IT Business and Cross Fog Applications: In a multivendor ecosystem applications need the ability to migrate and properly operate at any level of a fog deployment's hierarchy.

\section{QUANTUM COMPUTING}

\section{A. Concepts and Characteristics}

\section{1) Concepts}

Quantum computers are incredibly powerful machines that take a new approach to processing information. Built on the principles of quantum mechanics, they exploit complex and fascinating laws of nature that are always there, but usually remain hidden from view. By harnessing such natural behavior, quantum computing can run new types of algorithms to process information more holistically. They may one day lead to revolutionary breakthroughs in materials and drug discovery, the optimization of complex manmade systems, and artificial intelligence [5].

Quantum computing is a new type of calculation mode that follows the rules of quantum mechanics regulating quantum information units. The general theoretical model of a quantum computer is a universal Turing machine that is reinterpreted by using the laws of quantum mechanics. It can solve the problems that traditional computers can handle, and the computational efficiency is greatly improved due to the superposition of quantum mechanics. The research of quantum 
computing can be divided into computational model, software and hardware, and algorithm research [6].

2) Characteristics

Quantum computing has four main features:

- The only way to solve it is to guess answers repeatedly and check them.

- The number of possible answers to check is the same as the number of inputs.

- Every possible answer takes the same amount of time to check.

- There are no clues about which answers might be better: generating possibilities randomly is just as good as checking them in some special order.

\section{B. Development History}

The history of quantum computing is mainly from the theoretical stage to the commercial outbreak stage. It has gone through three stages, namely, the concept, the medium-term development and the prospect of development.

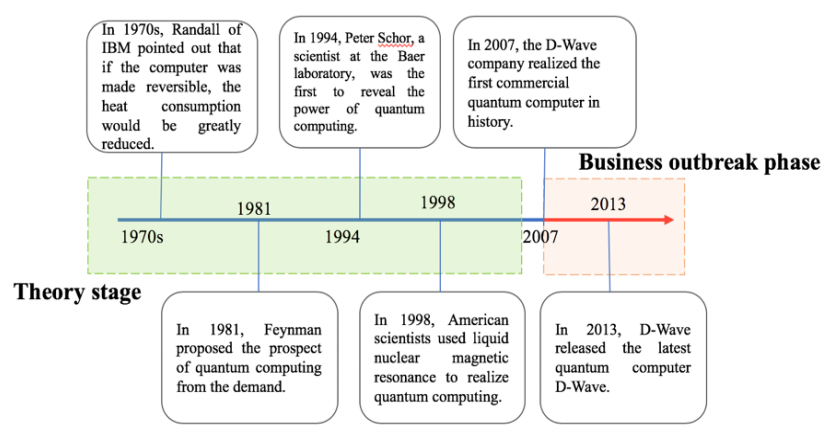

FIGURE II. DEVELOPMENT HISTORY OF QUANTUM COMPUTING

a) Concepts stage: The concept of quantum computing was first proposed by P. Benioff at the Argang National Laboratory in the early 1980 s.

b) Medium-term development: P. Shor, an applied mathematician at Baer laboratory, pointed out that quantum computing can break a large integer into the product of a qualitative factor with a shorter time in 1994.

c) Prospect of development: D-Wave, the Canadian quantum computing company, officially released the world's first commercial quantum computer D-Wave One in May 11, 2011.

\section{Related Technologies}

Quantum computer technology includes quantum hardware, quantum coding and quantum software. Quantum hardware is the core challenge of quantum computing.

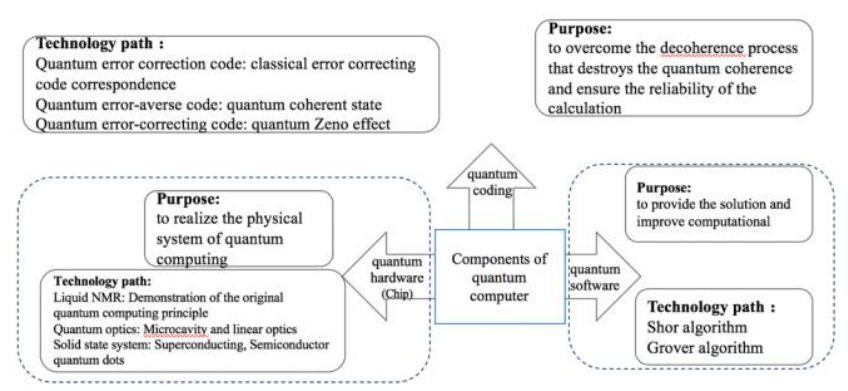

FIGURE III. RELATED TECHNOLOGIES OF QUANTUM COMPUTER

a) Quantum hardware: Quantum hardware is the main bottleneck for large-scale commercial applications of quantum. The main purpose of quantum hardware is to realize the physical system of quantum computing.

b) Quantum coding: The main purpose of quantum coding is to overcome the decoherence process that destroys the quantum coherence and ensure the reliability of the calculation.

c) Quantum software: Quantum software is mainly used to provide the solution and improve computational. The technology path includes Shor's algorithm and Grover's algorithm.

\section{Technical challenges}

There are a number of technical challenges in building a large-scale quantum computer, and thus far quantum computers have yet to solve a problem faster than a classical computer. IBM listed the following requirements for a practical quantum computer:

- Scalable physically to increase the number of qubits

- Qubits that can be initialized to arbitrary values

- Quantum gates that are faster than de-coherence time

- Universal gate set

- Qubits that can be read easily

\section{Cognitive COMPUTING}

\section{A. Definition}

Cognitive computing describes technology platforms that, broadly speaking, are based on the scientific disciplines of artificial intelligence and signal processing. These platforms encompass machine learning, reasoning, natural language processing, speech recognition and vision (object recognition), human-computer interaction, dialog and narrative generation, among other technologies [7].

\section{B. Features of Cognitive Computing}

The main features of cognitive computing include Iterative and stateful, adaptive, Interactive and contextual [8].

1) Iterative and stateful: They may aid in defining a problem by asking questions or finding additional source input if a problem statement is ambiguous or incomplete. 
2) Adaptive: They may learn as information changes, and as goals and requirements evolve.

3) Interactive: They may interact easily with users so that those users can define their needs comfortably.

4) Contextual: They may understand, identify, and extract contextual elements such as meaning, syntax, time, location, appropriate domain, regulations, user's profile, process, task and goal.

\section{Key Technologies}

- The top level technologies of cognitive computing is machine learning, natural language understanding and human-computer interaction.

- Big data technology, including the way to store, organize, manage and analyze.

- Computer architecture

- The bottom technologies of the cognitive computing needs atoms and nanoscale.

\section{UBIQUITOUS COMPUTING}

\section{A. Definition and Features}

Ubiquitous computing is a concept in software engineering and computer science where computing is made to appear anytime and everywhere, which can occur using any device, in any location, and in any format. The features of ubiquitous computing include Universality, transparency, dynamic, adaptive, diversity and eternity [9].

\section{B. Key Technolgoies}

The key technologies of ubiquitous computing include technical support, digital home and rebounding technology.

1) Technical support: Mobile communication technology, Global network services and P2P peer-to-peer computing.

2) Digital home: The digital home connects the broadband network to the family through the home gateway, as well as handheld devices, personal PC or household appliances that connect to the network in wired or wireless ways, which can provides a seamless, interactive and ubiquitous computing environment. People can access the community service network anytime and anywhere.

3) Rebounding technology: The rebounding technology developed by IBM is a thin screen board, which can display personal home page and custom content. People can work or send messages anywhere with a small card.

\section{INDUSTRIAL APPLICATIONS OF ADVANCED COMPUTING}

Advance computing can be widely used in aerospace, artificial intelligence, meteorological prediction and other industrial fields.
TABLE I. INDUSTRIAL APPLICATIONS OF ADVANCED COMPUTING

\begin{tabular}{|l|l|l|}
\hline NO. & CLASSIFICATION & \multicolumn{1}{|c|}{ INDUSTRIALAPPLICATIONS } \\
\hline 1 & Edge computing & $\begin{array}{l}\text { ladder network, smart grid, intelligent } \\
\text { street lamp, intelligent building, intelligent } \\
\text { IOV and intelligent agricultural machinery }\end{array}$ \\
\hline 2 & Fog computing & $\begin{array}{l}\text { Intelligent vehicle and traffic control, } \\
\text { Video security and monitoring program, } \\
\text { Intelligent city program }\end{array}$ \\
\hline 3 & Quantum computing & $\begin{array}{l}\text { Artificial intelligence, Machine learning, } \\
\text { Weather forecast, Traffic congestion and } \\
\text { Aerospace }\end{array}$ \\
\hline 4 & Cognitive computing & $\begin{array}{l}\text { self-driving cars, Automatic data input and } \\
\text { automatic handwriting recognition and } \\
\text { machine learning }\end{array}$ \\
\hline 5 & Ubiquitous computing & Grid computing and Bluetooth \\
\hline
\end{tabular}

\section{CONCLUSION}

Advanced computing is the key technical means to solve the major challenges of national economic construction, social development, scientific progress and national security, which is an important part of the national innovation system.

At present, the United States, Japan and the EU have invested a large amount of capital and manpower in the field of advanced computing technology to ensure the leadership of the technology. China has also issued relevant policies to support the development of advanced computing and industrial applications.

We propose to start the research on the topic of Standardization requirements analysis on advanced computing. Since Advanced Computing is useful for IoT, AI, Big data and other IT applications, it is necessary to set up a new working group on Advanced Computing.

\section{REFERENCES}

[1] Third International Conference on Computing Communication \& NETWORKING Technologies. IEEE, 2012:1-5.

[2] Kirkland E J. Advanced Computing in Electron Microscopy[M]. Springer US, 2010.

[3] Satyanarayanan M. The Emergence of Edge Computing[J]. Computer, 2017, 50(1):30-39.

[4] Bonomi F, Milito R, Zhu J, et al. Fog computing and its role in the internet of things[C], 2012:13-16.

[5] Leuenberger M N, Loss D. Quantum computing in molecular magnets[J]. Nature, 2001, 410(6830):789-93.

[6] Stepanenko D, Trif M, Loss D. Quantum computing with molecular magnets[J]. Inorganica Chimica Acta, 2008, 361(14):3740-3745.

[7] Wang Y, Baciu G, Yao Y, et al. Advances in Cognitive Informatics and Cognitive Computing[J]. Studies in Computational Intelligence, 2009, 4(4):9-27.

[8] Preissl R, Wong T M, Datta P, et al. Compass: A scalable simulator for an architecture for cognitive computing[C]// High PERFORMANCE Computing, Networking, Storage and Analysis. IEEE, 2012:1-11.

[9] Ranganathan A, Campbell R H. A Middleware for Context-Aware Agents in Ubiquitous Computing Environments[C], 2014:143-161. 